\title{
Design and Performance Analysis of Compact Wearable Textile Antennas for IoT and Body-Centric Communication Applications
}

\author{
Sanjit Varma $\left(\mathbb{D},{ }^{1}\right.$ Somia Sharma $\mathbb{D}^{2}{ }^{2}$ Merbin John $\mathbb{D}^{1},{ }^{1}$ Richa Bharadwaj $\left(\mathbb{D},{ }^{2}\right.$ \\ Anuj Dhawan $\mathbb{D}^{1},{ }^{1}$ and Shiban K. Koul $\mathbb{D}^{2}$ \\ ${ }^{1}$ Department of Electrical Engineering, IIT Delhi, New Delhi 110016, India \\ ${ }^{2}$ Centre for Applied Research in Electronics, IIT Delhi, New Delhi 110016, India \\ Correspondence should be addressed to Richa Bharadwaj; richabharadwaj@hotmail.com
}

Received 8 April 2021; Revised 23 July 2021; Accepted 12 August 2021; Published 24 August 2021

Academic Editor: Sachin Kumar

Copyright (C) 2021 Sanjit Varma et al. This is an open access article distributed under the Creative Commons Attribution License, which permits unrestricted use, distribution, and reproduction in any medium, provided the original work is properly cited.

\begin{abstract}
This paper presents two compact textile-based planar dipole and loop antennas for wearable communication applications operating in the $2.4 \mathrm{GHz}$ industrial, scientific, and medical radio (ISM) bands. The antennas were fabricated on a $0.44 \mathrm{~mm}$ thin camouflaged-military print, cotton jean cloth using conductive copper threads, and sewing embroidery technique to create the radiating structure. Design and performance analyses of the antennas were carried out using simulations; further experiments were performed in anechoic chamber and indoor environment to validate the designs. The experiments were carried out in a free space scenario and on the various locations of the human subject such as the torso and limb joints. The performance of the antennas was investigated based on the reflection coefficient in normal and bent conditions corresponding to the different radii of the locations of the human limbs. The antennas perform well in free space and on-body scenarios in flat and bend conditions providing return loss below $-10 \mathrm{~dB}$ in all cases with an acceptable resonant frequency close to $2.4 \mathrm{GHz}$ due to the antenna bending and body effects. The radiation pattern measurements are also reported in this work for free space and on-body scenarios. It is observed that the presence of the human body significantly influences the antenna radiation pattern which leads to an increase in the front-to-back ratio and also makes the antenna more directive. Overall, the performance of the fabricated embroidered textile antennas was found suitable for various wearable body-centric applications in indoor environments.
\end{abstract}

\section{Introduction}

Wearable communication technologies offer promising solutions for applications in the field of biomedical, consumer electronics, sports, military, and smart home applications. Recent developments in miniaturized and flexible electronic devices have made commercialization of such devices possible which has paved the road for vast utility in wearable Internet of Things (IoT) applications [1]. Their lightweight, low-cost manufacturing, ease of fabrication, and the availability of inexpensive flexible substrates (i.e., papers, textiles, and polymers) make flexible electronics an appealing candidate for the next generation of consumer electronics $[2,3]$.

Research is being carried out at various frequency bands available in the open literature for wireless body area network (WBAN) applications ranging from $2.4 / 5.8 \mathrm{GHz}$ industrial, scientific, and medical (ISM) band [4], 3.1-10.6 GHz ultra-wideband (UWB) [5-7], and the millimetere wave (mm-Wave) band $57-64 \mathrm{GHz}[8,9]$. The $2.4 \mathrm{GHz}$ ISM band is very suitable for wearable body-centric communication due to the global availability of the spectrum and wide range of applications in the field of healthcare, smart homes, sports, military, and day-to-day life $[2,3]$.

Antenna is one of the key components of the wearable communication device providing a robust communication link between various devices. Wearable antennas should be very compact, low-profile, lightweight, mechanically robust, efficient, and preferably flexible to suit the conformal structure of the body surface [2-5]. These antennas should also perform well when placed in proximity of the human body and have suitable performance in terms of operating 
frequency and desired radiation characteristics [10]. Several works have been carried out on rigid substrates which lack flexibility and can be fragile due to the dynamic movements and postures made by the user. To address this problem, various flexible substrates are proposed in the open literature. Commonly used flexible materials for antennas are polyimides (PI), polydimethylsiloxane (PDMS), polytetrafluoroethylene (PTFE), liquid crystal polymer (LCP), Kapton, and paper-based substrates $[2,3,6-9,11]$.

Apart from polymer-based substrates, textile-based substrates are also gaining popularity due to the ease of integration of the antenna directly with the clothing as well as due to the flexible and conformal nature of the textile fabrics [12]. Textile materials commonly used as substrates are cotton cloth, felt, denim, fleece, nylon, and polyester. Different fabrication methods are chosen depending on the substrate material, application, and suitability; these methods include inkjet-printing, screen-printing, 3D printing, sewing, and embroidered techniques $[2,3,13,14]$. Textile material selection in terms of electrical properties and thickness, manufacturing process, feeding method, and the overall antenna design are important aspects to be considered while designing such antennas. Antenna designs such as dipole, loop, spiral, and patch are commonly chosen structures for various communication applications [15-20]. Advanced antenna structures like (electromagnetic bandgap) EBG, artificial magnetic conductors (ACM), and substrate-integrated waveguide (SIW) antennas to enhance the performance of the antenna have been reported in open literature [21-23].

This paper proposes two compact, low-profile textilebased antennas which can be integrated into the clothing suitable for healthcare, indoor, and military IoT applications. The proposed design exploits the lightweight and flexible properties of the cotton cloth along with the high conductivity copper threads which are embroidered using sewing technology to form the radiating elements over the textile. The dipole antenna operates at $2.19-3.44 \mathrm{GHz}$ and the loop antenna operates at $2.35-2.81 \mathrm{GHz}$ frequency range in free space scenario. Various antenna parameters such as return loss and radiation patterns have been analysed for free space and on-body scenarios. Flexibility tests have also been carried out by bending the antenna at different radii corresponding to that of the radii of the limb joints of the human subject.

The novelty of the work described in this paper lies in the fabrication of highly conformal and flexible textile antennas using highly flexible customized copper threads in an automated manner. Moreover, this paper presents an in-depth analysis and comparison for the two textile antennas for onbody measurements in flat as well as bending scenarios (apart from free space scenarios), which is limited in the open literature. Various positions of the limbs have also been analysed to see the effect of orientation and proximity with the torso/thigh region depending on the antenna location. a $0.44 \mathrm{~mm}$ thin jeans cloth has been employed as a substrate which makes the antenna design more challenging.

The antenna prototypes are presented in Section 2 along with the simulation and measurement results. Section 3 gives details of the on-body measurement procedure and compares free space and on-body measurements related to $S_{11}$ and far-field radiation patterns. Section 4 deals with the study on the influence of the antenna performance when bent at different radii for free space and on-body scenarios and key findings are reported. The conclusion is presented in Section 5 with highlights on the future aspects.

\section{Fabricated Textile Antenna Prototype and Performance Analysis}

The proposed textile dipole and loop antenna structures comprise the radiating elements (two arms and rectangular loop structure, resp.) fabricated using the copper metal threads over a jeans cotton cloth fabric. These flexible and conformal copper threads or yarns were developed by twisting multiple thin copper wires, each wire's diameter being $0.071 \mathrm{~mm}$. The antennas are designed to work at $2.4 \mathrm{GHz}$, ISM band using the jeans cotton material as the substrate with a relative permittivity of 1.67 [12]. The copper threads are embroidered into the substrate material of the antennas to form the radiating elements. The thickness of the copper thread is $0.345 \mathrm{~mm}$ throughout and the substrate thickness is around $0.44 \mathrm{~mm}$.

The final schematic and dimensions of the antennas are present in Figure 1(a) for the dipole antenna and Figure 1(b) for the loop antenna. The length of the dipole antenna arm is calculated by half-wavelength of the desired frequency, that is, $2.4 \mathrm{GHz}$. The separation between the two antenna arms and the length of those arms are important factors that are sensitive to the change in the output frequency at which the antenna performs. To verify the proposed design performance before physically fabricating them using the embroidery sewing tool, a CST model was simulated with the same dimensions.

The sewing machine used to fabricate the copper-threadbased antennas over the textile material is Bernina B720 [24]. This machine has a Bernina hook system, which gives it a maximum sewing and embroidery speed of 1000 stitches per minute. The maximum stitch width the tool can provide is $5.5 \mathrm{~mm}$ with a maximum stitch length of $6 \mathrm{~mm}$. The antenna design models were integrated with the tool using the "Bernina Embroidery Software Designer Plus" software which was purchased along with the sewing tool itself. Figure 1(c) shows the sewing process in which a dipole antenna pattern is being embroidered using copper thread over the denim cloth fabric.

A thin $3 \mathrm{~mm}$ foam (which provides no changes in the relative permittivity) was applied on the backside of the antenna fabric to add extra support to the textile during free space and body-centric measurements. The fabricated prototype of the textile dipole antenna and loop antenna is presented in Figures 1(d) and 1(e), respectively. A $50 \Omega$ SMA connector was carefully soldered to the edges of the dipole and rectangular loop structures.

The performance of the wearable textile antennas was measured through a programmable 2-port vector network analyser (PNA-E8364C) in terms of reflection coefficient $\left(\mathrm{S}_{11}\right)$ and compared with the simulated results obtained from CST 


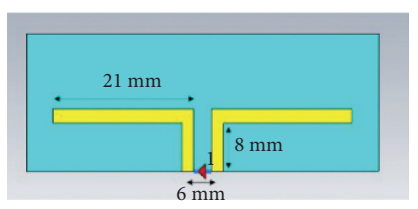

(a)

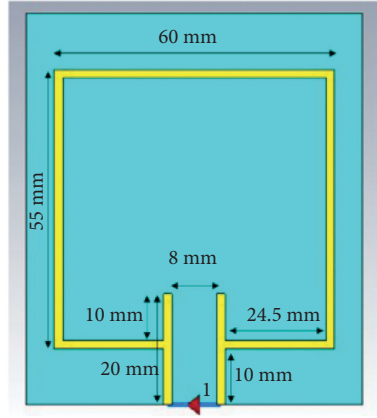

(b)

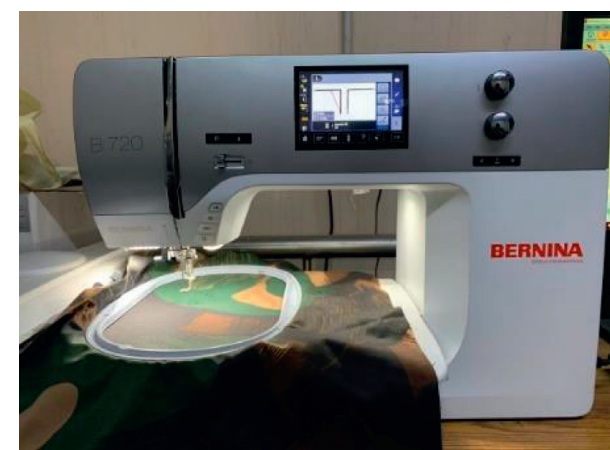

(c)

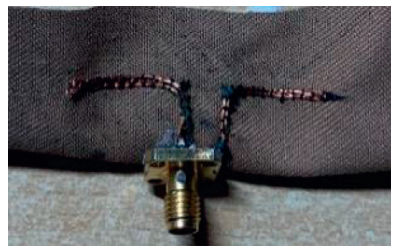

(d)

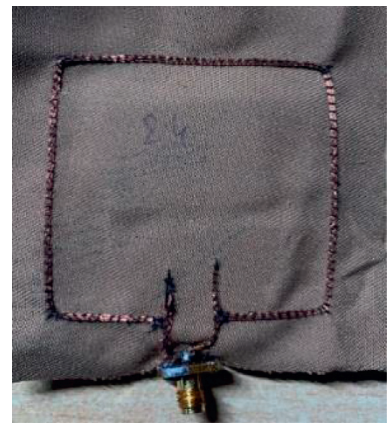

(e)

Figure 1: Schematic of the textile-based (a) dipole and (b) loop antenna. (c) The Bernina 720 sewing machine tool used for sewing the radiating element structures in the form of embroidered pattern over the denim-cotton fabric. Fabricated prototype of the textile (d) dipole and (e) loop antenna.

microwave studio. Radiation pattern measurements were also carried out in the anechoic chamber at the RF and Microwave Lab, CARE, IIT Delhi, and with those obtained from the simulation results. Good agreement is found between the simulation and measurement results for both proposed antennas. Figure 2(a) presents $S_{11}$ results for the simulated and measured textile dipole antenna structure. It is observed that both results provide below $-10 \mathrm{~dB}$ return loss and resonate in the $2.4 \mathrm{GHz}$, ISM band, and perform well in a free space/indoor environment. The deviations observed in the simulated and measured results can be possibly attributed to the radiating material in these textile antennas being conductive threads in comparison to a uniform sheet of copper in the simulated design, as well as to a difference in the permittivity of the substrate employed in the simulations from that of the jeans cloth substrate in these antennas. The presence of a $50-\mathrm{ohm}$ SMA conductor soldered to the antenna structure can also bring about some variation between the measured and simulated results. Figures 2(b) and 2(c) present the simulated and measured radiation pattern of the dipole antenna for E-plane and H-plane. Similar radiation patterns are observed for both cases, hence validating the performance of the fabricated antenna.

\section{Body-Centric Measurement Scenarios}

Body-centric measurements were performed on a human subject in an indoor laboratory environment in the $2.4 \mathrm{GHz}$ ISM frequency band. The male human subject has a height of $167 \mathrm{~cm}$ and a weight of $74 \mathrm{~kg}$ with an average build. The wearable antenna was placed on the torso region of the human subject to compare with the free space $S_{11}$ results (Figure $3(\mathrm{a})$ ). The wearable textile antennas are able to achieve below $-10 \mathrm{~dB}$ return loss for onbody scenarios indicating good performance of the antenna when placed in the proximity of the body. An example of the measured and simulated $S_{11}$ results of the textile loop antenna is presented in Figure 3(b), for both the free space and the on-body scenarios. For the on-body simulation results, a phantom representing the torso region of the human body with dimensions $200 \times 200 \times 70 \mathrm{~mm}^{3}$ is designed in CST microwave studio. The human phantom consists of skin, fat, and muscle as the three layers with electrical properties such as permittivity and conductivity as per data provided in [25] for $2.4 \mathrm{GHz}$ frequency. The thickness of the skin, fat, and muscle layer is $3 \mathrm{~mm}, 7 \mathrm{~mm}$, and $60 \mathrm{~mm}$, respectively [26]. A shift of $100 \mathrm{MHz}$ in the resonant frequency is observed when placed over the body $(2.4 \mathrm{GHz})$ in comparison to the free space $(2.5 \mathrm{GHz})$ results which is due to the body effects that influence the antenna performance.

Apart from $S_{11}$ measurements, far-field radiation pattern measurements are also carried out for both antennas studied in an anechoic chamber for free space and on-body scenarios. The antenna pattern measurements are performed for E-plane and H-plane to understand the influence of the human subject in both planes. The results for the textile dipole antenna and loop antenna are presented in 


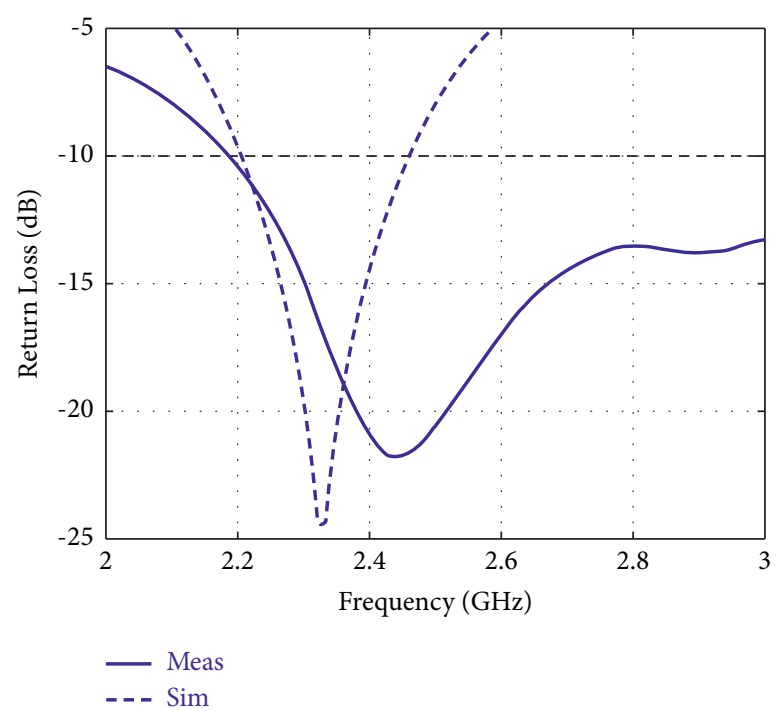

(a)

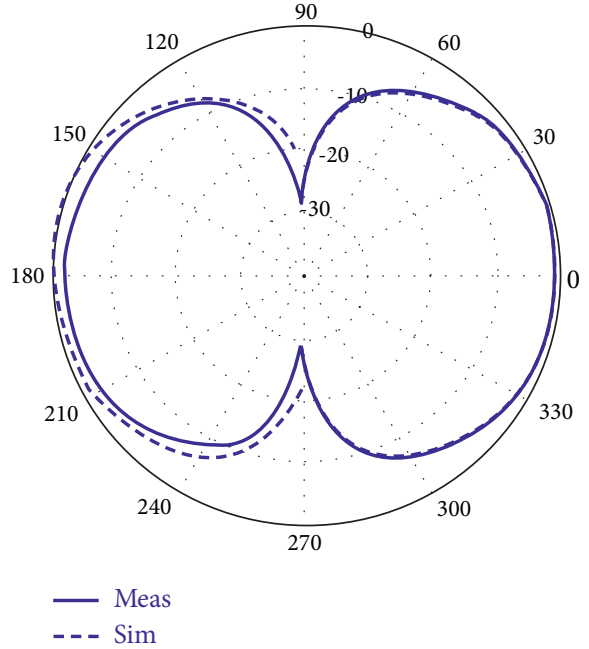

(b)

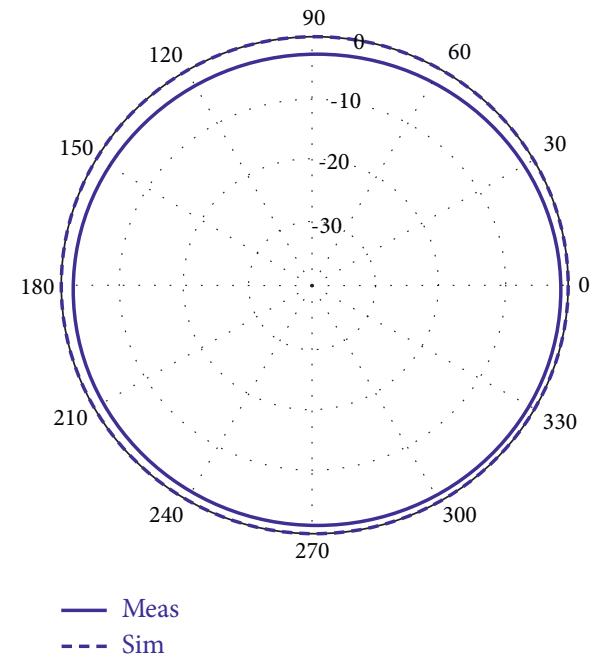

(c)

Figure 2: Measured and simulation results for $S_{11}$ parameters for free space scenario: (a) dipole antenna. Measured and simulated antenna radiation pattern results: (b) E-plane and (c) H-plane.

Figures 4(a)-4(d), respectively. It is observed that both antennas are strongly influenced by the presence of the human subject which increases the front-to-back ratio of the radiation pattern and makes the pattern more directive in nature $[27,28]$.

\section{Impact of Antenna Bending}

One of the main challenges of the textile wearable antenna design is the uncertain form of the garment surface due to frequent movements performed by the human subject and also the nature of the fabric [29]. Hence, the wearable antennas integrated with the garment also encounter scenarios such as bending, stretching, flexing, and crumpling apart from the normal flat condition $[29,30]$. This section evaluates the antenna performance under bending conditions for free space and on-body scenarios.
4.1. Free Space Measurements. For free space measurements, the antenna is bent over a cylindrical foam of different radii corresponding to the curvatures of the joints of the limbs of the human subject. The limb joints considered are wrist, elbow, shoulder, and knee. Figures 5(a) and 5(b) present the experimental setup for the antenna bent on foam for the dipole antenna for the radii corresponding to the wrist and elbow. Figures 5(c) and 5(d) present the experimental setup for the antenna bent on foam for the loop antenna for the radii corresponding to the shoulder and knee. Table 1 summarises the radii and circumference values for various limb locations chosen that are considered for free space foam experiments to measure the $S_{11}$ parameters.

The $S_{11}$ results corresponding to different bending radii are presented in Figures 6(a) and 6(b) for the dipole and loop antenna, respectively. It is observed that the bending condition of the textile antenna has a strong effect on the $S_{11}$ 

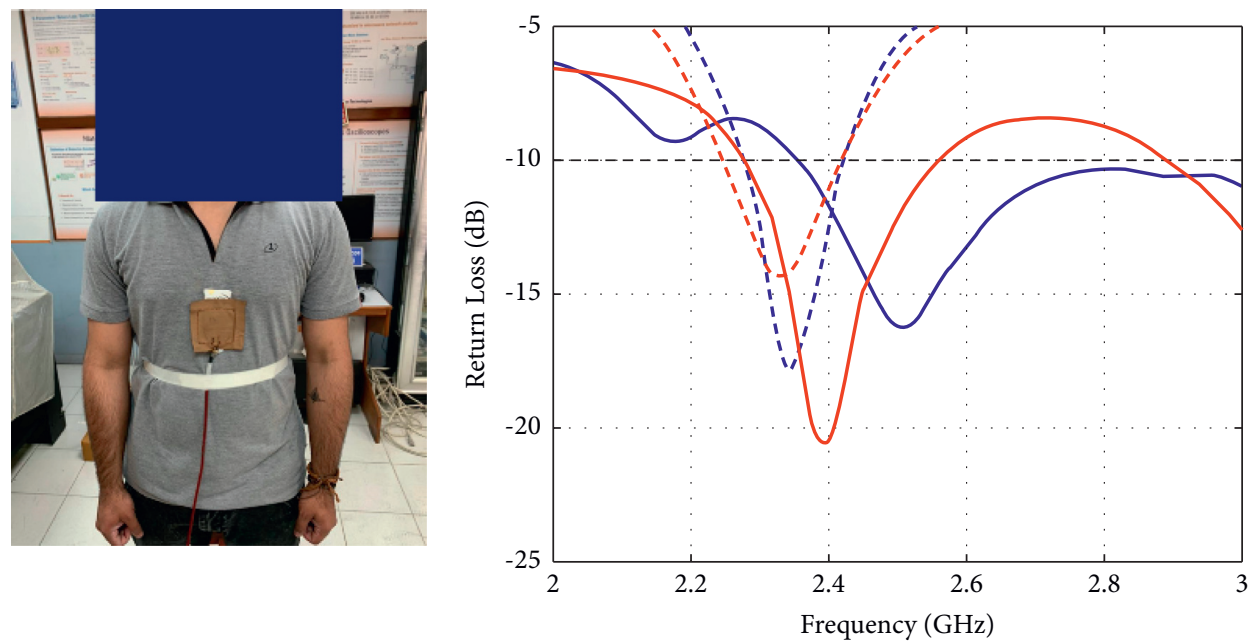

- Meas FS

- Meas FS On-B

- - - Sim FS

- - - Sim FS On-B

(a)

(b)

Figure 3: (a) Textile loop antenna is placed on the torso region of the human subject. (b) Free space (FS) and on-body (On-B) $\mathrm{S}_{11}$ measurement and simulation results.
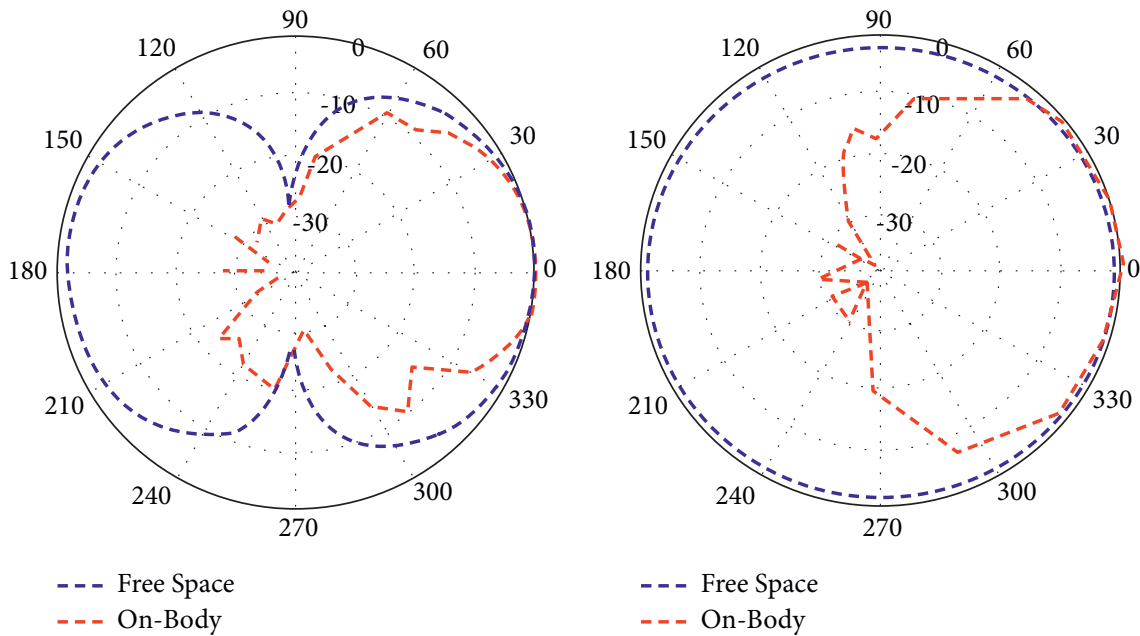

(a)

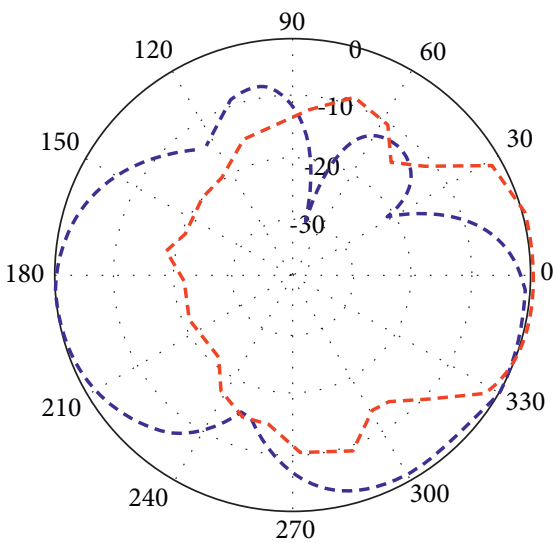

(b)

- - - Free Space

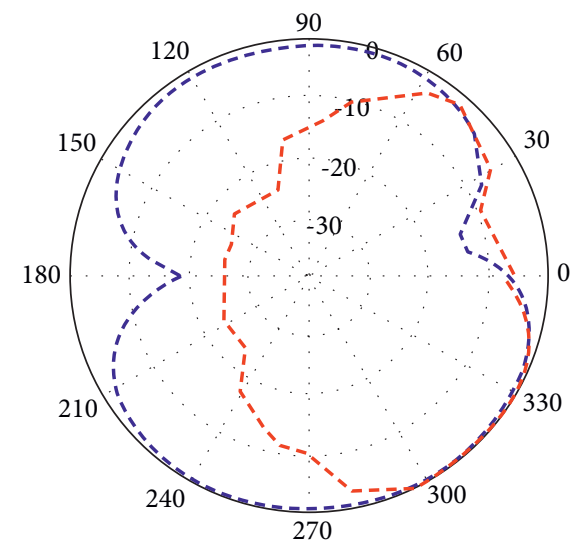

- - - Free Space

- - - On-Body

(c)

(d)

Figure 4: Measured free space and on-body radiation patterns. Dipole antenna: (a) E-plane and (b) H-plane. Loop antenna: (c) E-plane and (d) H-plane. 


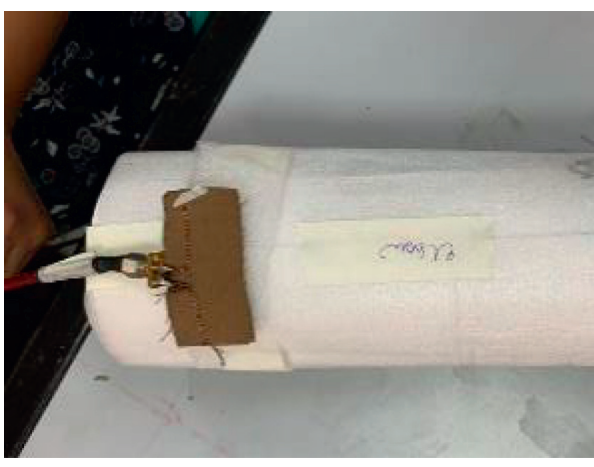

(a)

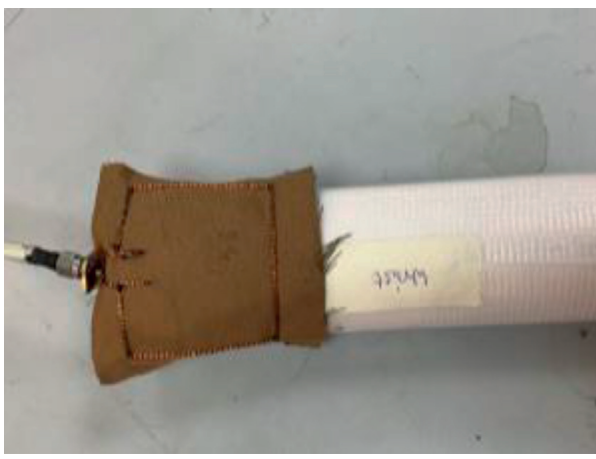

(c)

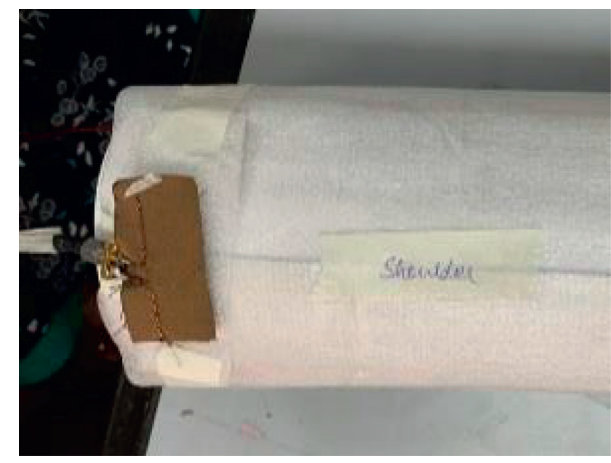

(b)

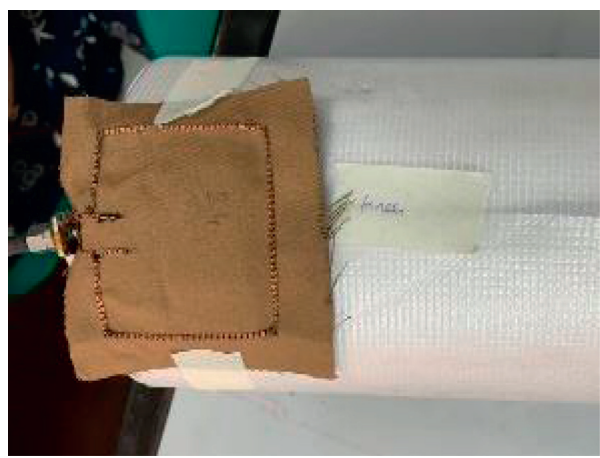

(d)

Figure 5: Photograph of the various antenna structures bent with different radii corresponding to the limb joint locations. Textile dipole antenna: (a) elbow and (b) shoulder. Textile loop antenna: (c) wrist and (d) knee.

TABLE 1: Wearable antenna locations: radii values for free space and on-body antenna bending experiment

\begin{tabular}{lcc}
\hline & Radii $(\mathrm{mm})$ & Circumference $(\mathrm{mm})$ \\
\hline Upper limbs & & \\
Wrist & 25.7 & 161.5 \\
Elbow & 42.9 & 270.0 \\
Shoulder & 54.1 & 340.0 \\
\hline Lower limbs & & \\
Knee & 57.2 & 359.4 \\
\hline
\end{tabular}

results leading to a shift in the resonant frequency and also the magnitude of the $S_{11}$ results. Significant deviation from the flat case results is observed for the lowest bending radii $=25.7 \mathrm{~mm}$ as the antenna structure is quite affected by the degree of bending taking place especially for the textile dipole antenna. The bending scenario leads to a change in the resonant length of the antenna leading to variation in the $\mathrm{S}_{11}$ characteristics. Minimum variation is observed for the highest bending radii $=57.2 \mathrm{~mm}$ as this scenario is approaching towards the flat orientation of the antenna. As observed in Figure 6(a), the dipole antenna resonant frequency spans from $2.45 \mathrm{GHz}$ to $2.58 \mathrm{GHz}$ and the loop antenna resonant frequency spans from $2.48 \mathrm{GHz}$ to $2.52 \mathrm{GHz}$ for the bending radii range considered. The $\mathrm{S}_{11}$ magnitude of the resonant frequency varies from $-20.16 \mathrm{~dB}$ to $-18.56 \mathrm{~dB}$ and -15.41 to $-14.98 \mathrm{~dB}$ for the dipole and loop antenna, respectively. The variation of the resonant frequency and the magnitude is more significant for the textile dipole antenna.
4.2. On-Body Measurements. On-body antenna bending experiments have been carried out for various locations of the human subject which are the wrist, elbow, shoulder, and knee joints. The antenna bending experiment is performed for two positions of the upper and lower limb while the subject is in a sitting posture. In the first position, the arm is at a 0 -degree position with respect to the shoulder joint (as depicted in Figure 7(a)) and the leg is stretched out straight making 0 degrees with respect to the thigh joint (as depicted in Figure 7(b). In the second scenario, the position of the arm is at 90 degrees with respect to the shoulder joint (as depicted in Figure 7(c)) and the leg bent at 90 degrees with respect to the knee joint (as depicted in Figure $7(\mathrm{~d})$ ).

As observed in Figures 8(a), 8(b), 9(a), and 9(b) corresponding to the dipole antenna and loop antenna, respectively, the $S_{11}$ magnitude and resonant frequency vary for various on-body locations on the antenna. This is attributed to the body effects and also the different curvatures of bending of the textile antenna depending on the radii of 


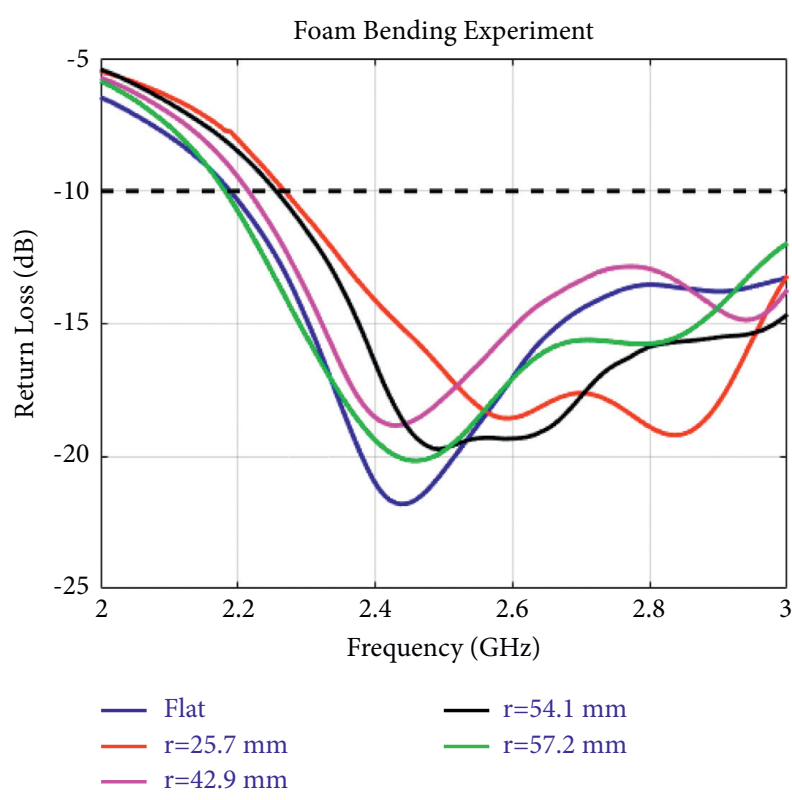

(a)

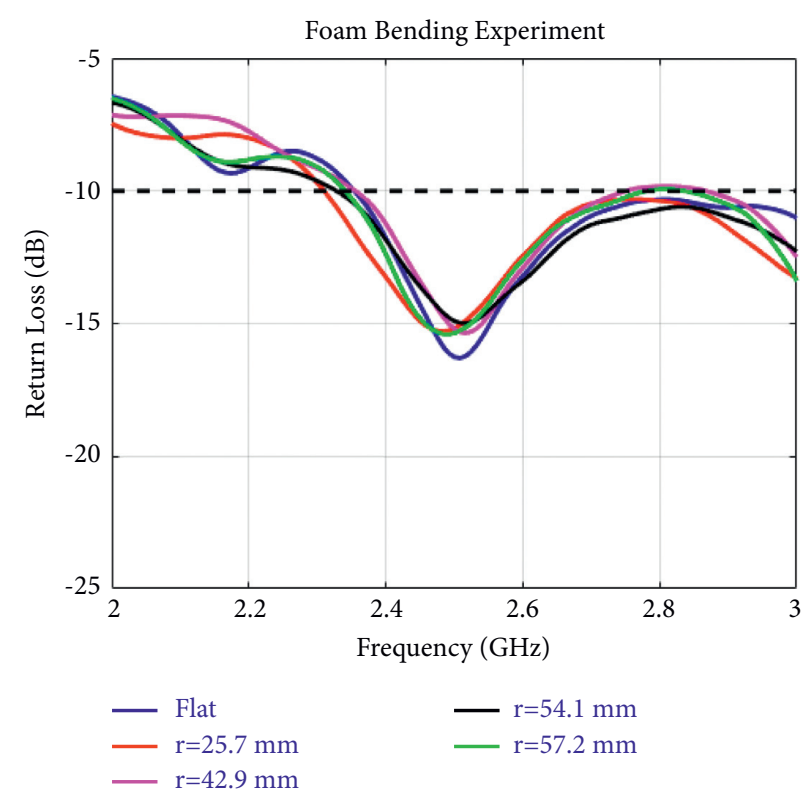

(b)

FIgURE 6: Measured $S_{11}$ plots of the antennas bent with different radii: (a) dipole antenna and (b) loop antenna. Bending radii considered ranges from $25.7 \mathrm{~mm}$ to $57.2 \mathrm{~mm}$ and compared with the flat case scenario.

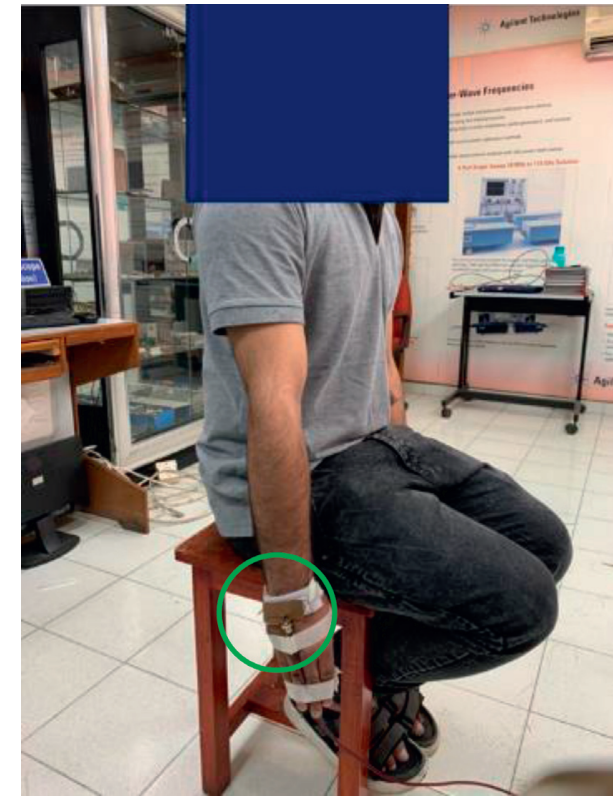

(a)

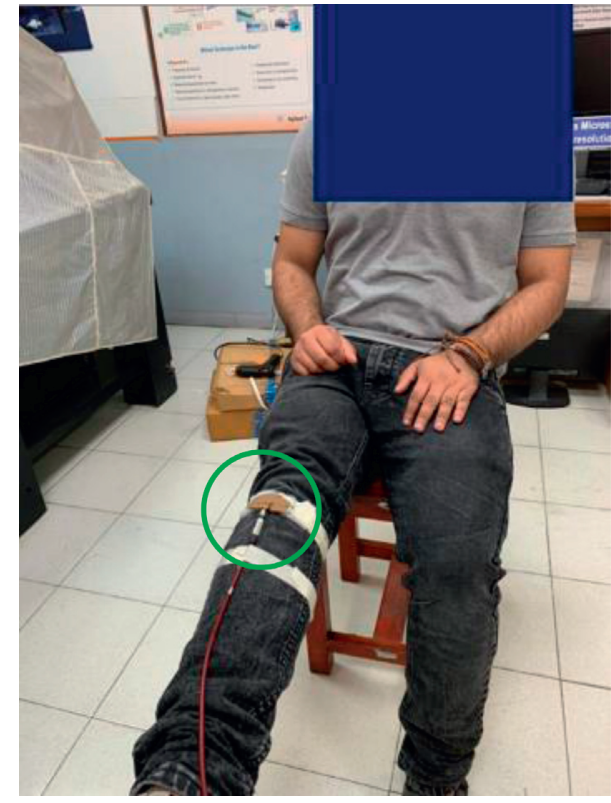

(b)

Figure 7: Continued. 


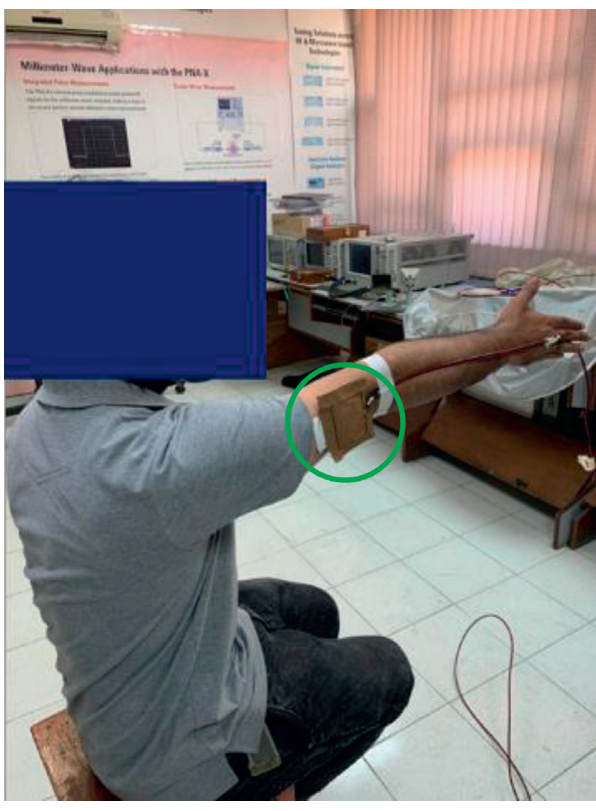

(c)

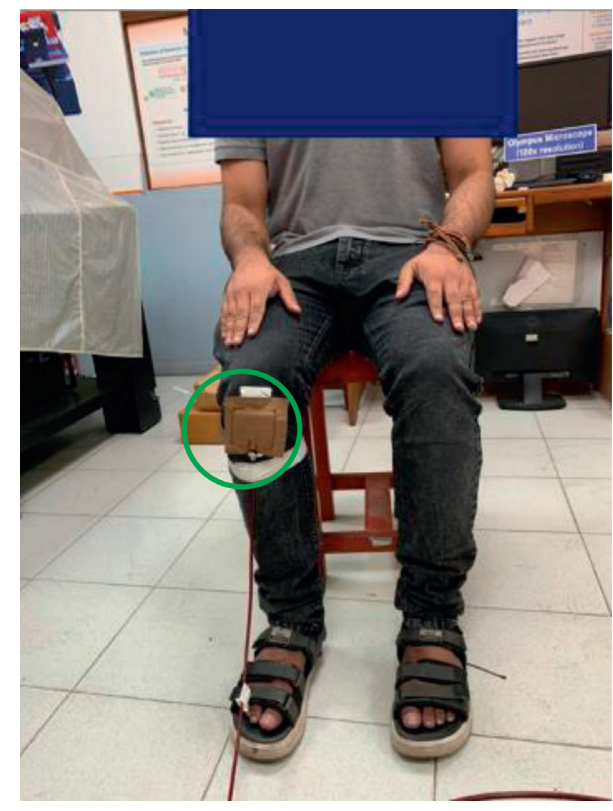

(d)

Figure 7: Measurements for various on-body antenna locations and bending curvatures for the upper and lower limbs. Position I (0 degrees): (a) dipole antenna, wrist, and (b) dipole antenna, knee. Position II (90 degrees): (c) loop antenna, elbow, and (d) loop antenna, knee.

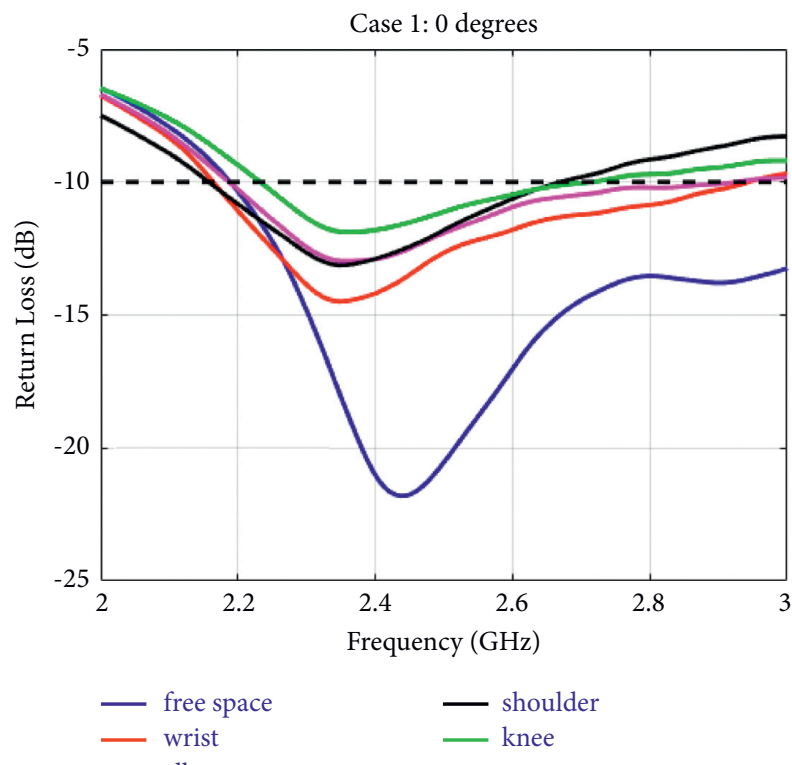

(a)

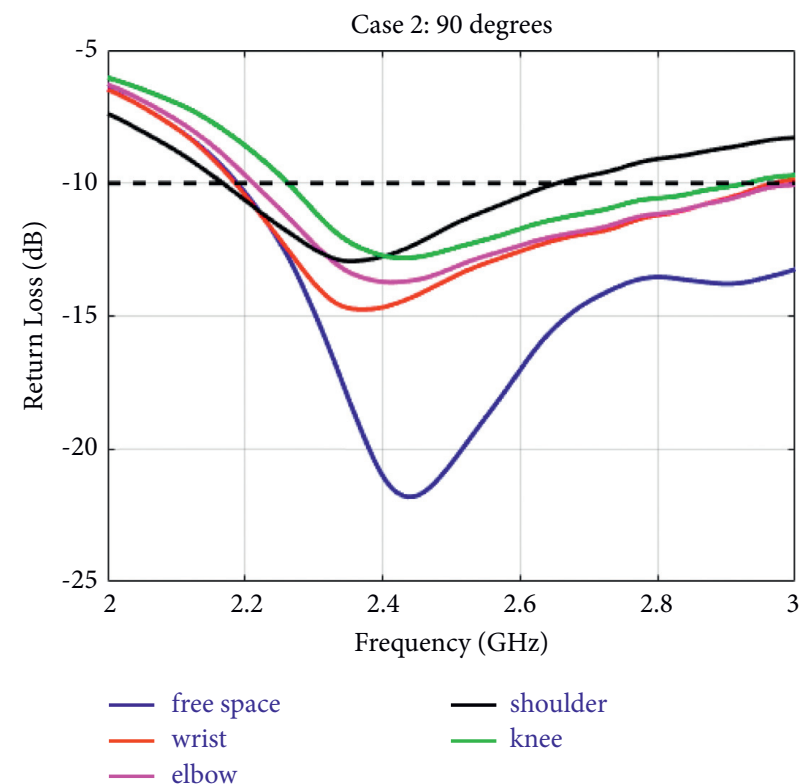

(b)

FIgURE 8: Variation in $S_{11}$ results for the textile dipole antenna for various on-body limb locations and bending radii: (a) position I (0 degrees) and (b) position II (90 degrees).

the body locations. For the dipole antenna, the $S_{11}$ curve shifts upward due to the antenna bending with a span of magnitude ranging from $-14.5 \mathrm{~dB}$ to $-11.8 \mathrm{~dB}$ for Position I and $-14.9 \mathrm{~dB}$ to $-12.8 \mathrm{~dB}$ for Position II. The resonant frequencies shift in the range of $2.34 \mathrm{GHz}$ to $2.38 \mathrm{GHz}$ for Position I and $2.38 \mathrm{GHz}$ to $2.41 \mathrm{GHz}$ for Position II. Both orientations of the limbs 0 and 90 degrees show similar variation in the $S_{11}$ parameters with a 90-degree scenario depicting slightly better $S_{11}$ resonance. This can be due to the reduction in body effects caused from the torso or thigh region when the limbs are positioned in the 90-degree case.

For the textile loop antenna, higher fluctuation in the magnitude of the $S_{11}$ results are observed (Figures 9(a)-9(b)) for the different on-body locations and bending curvatures. 


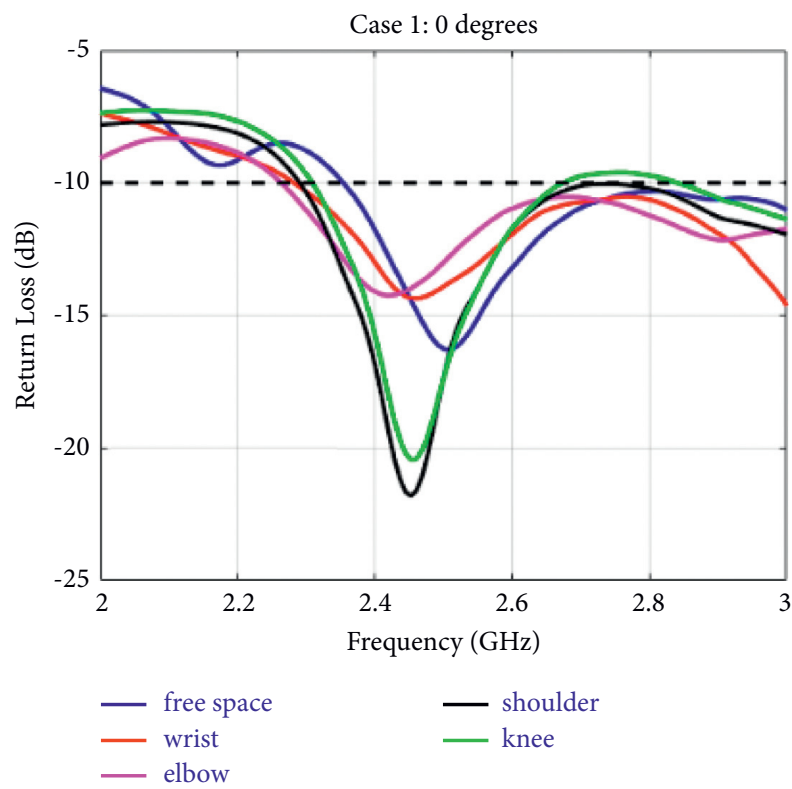

(a)

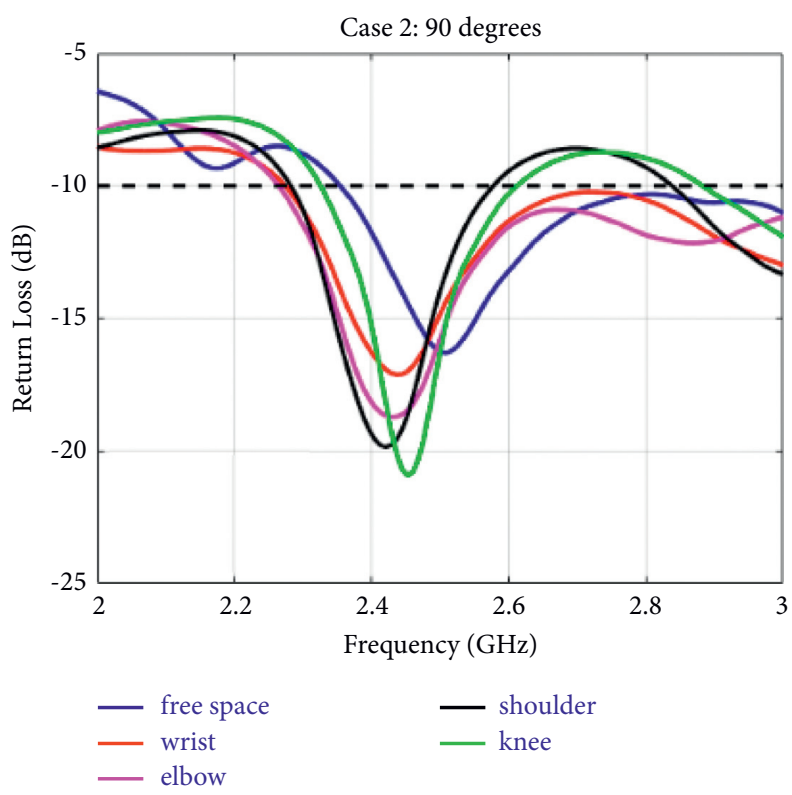

(b)

FIGURE 9: Variation in $\mathrm{S}_{11}$ results for the textile loop antenna for various on-body limb locations and bending radii: (a) position I (0 degrees) and (b) position II (90 degrees).

TABLe 2: Comparison of textile antennas with proposed antennas.

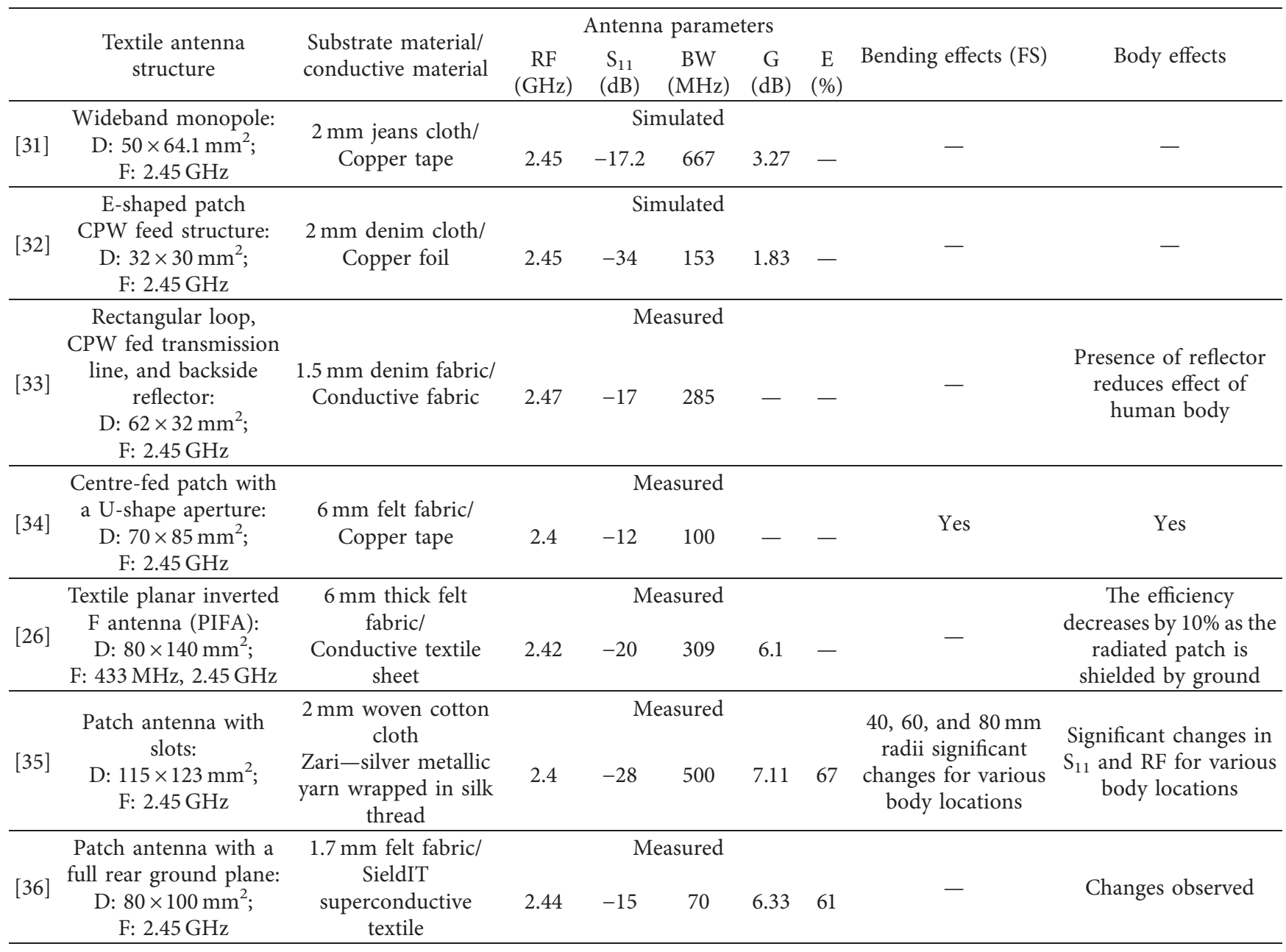


TABle 2: Continued.

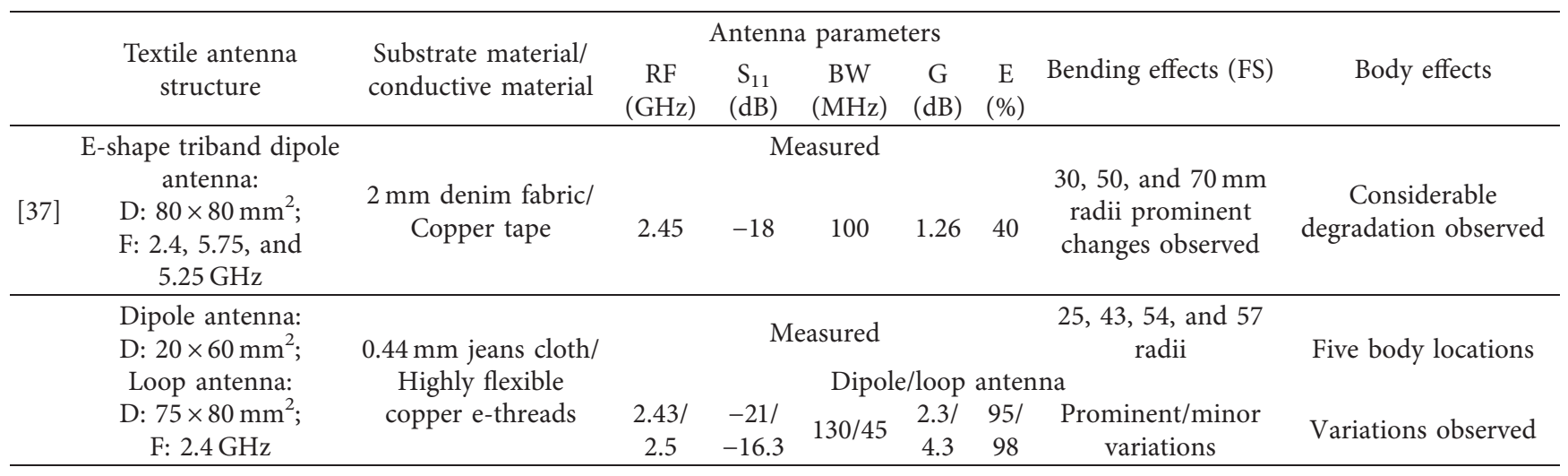

The magnitude varies from $-21 \mathrm{~dB}$ to $-14 \mathrm{~dB}$ for the various on-body locations considered. This variation depends on various factors such as the bending radii, surface on which the antenna is placed on the body (directly on the skin/above clothing) and distance from the body surface. The shift in the resonant frequency from the free space results is less for the various bending radii chosen in comparison to that of the textile dipole antenna.

The shift ranges from $2.42 \mathrm{GHz}$ to $2.45 \mathrm{GHz}$ of frequency. It can be concluded that the type of antenna, location of the wearable antenna, bending radii of the antenna, and also the orientation of the limbs can cause some variation in the $S_{11}$ characteristics such as resonant frequency, impedance bandwidth, and $S_{11}$ magnitude. It is also observed that, in all the scenarios considered, the magnitude of the $S_{11}$ is below $-10 \mathrm{~dB}$ and also resonating close to the desired frequency of operation which is the $2.4 \mathrm{GHz}$ ISM band. For the dipole antenna, the shift observed ranges from $10 \mathrm{MHz}$ to $60 \mathrm{MHz}$ and for the loop antenna, the shift observed is in the range of $20 \mathrm{MHz}$ to $50 \mathrm{MHz}$, for on-body scenarios when compared with the desired $2.4 \mathrm{GHz}$ resonant frequency.

\section{Comparison with Related Previous Works}

Table 2 compares the work described in this paper with the other existing works from the literature [26, 31-37]. The novelty of the work described in this paper lies in the fabrication of highly conformal, flexible, and compact textile antennas using highly flexible customized copper threads in an automated manner. The fabrication of these low-profile textile antennas was carried out on very thin textile substrates that are suitable for free space and body-centric applications. The performance of the two antennas is compared for various bending conditions and on-body scenarios for which a detailed analysis has been provided. It has to be mentioned that such comparison or analysis is very limited considering the research work on textile antennas present in the open literature. The body-centric measurements have been carried out for various locations on the body and also for different orientations of the limbs. The proposed antennas have been designed using a very thin textile jean cloth (of thickness $0.44 \mathrm{~mm}$ ) and are still able to achieve the desired resonant frequency in normal conditions as well as in bending scenarios. The proposed antennas are fabricated on the jeans cloth using digital embroidery technology with the radiating elements made from highly conformal and flexible customized copper e-threads of diameter $0.345 \mathrm{~mm}$, which gives acceptable results for various on-body locations and limb orientations. The copper yarn is made from twisting multiple copper threads of $0.071 \mathrm{~mm}$ in diameter. The copper e-threads have higher conductivity and provide higher performance in comparison to threads made of other metals (such as silver or steel) that have been employed in previous works $[14,35,38]$. Most of the works in open literature use conductive fabrics $[26,33,36]$ or copper tapes/foil [31, 32, 34, 37] which are glued over the textile fabric that makes the antenna not durable to withstand repeated use and harsh environments. The use of e-threads (conductive threads) as the radiating elements makes it feasible to integrate the antenna into clothing, thus making the movement of human subject unobtrusive and comfortable.

\section{Conclusion}

This work proposes two textile-based antennas for IoT and body-centric communication applications, namely, dipole and loop antenna. The performance of the wearable antenna is investigated in free space and in proximity with the human body. The antennas have been fabricated on cost-effective and commercially available textile cotton cloth and the radiating structure made of conductive copper threads has been formed using the sewing embroidered technique. The proposed antennas are compact and flexible with good agreement between the measured and simulated results operating at $2.4 \mathrm{GHz}$.

Return loss and radiation pattern characteristics for free space and on-body scenarios have been presented and discussed. The return loss of the antenna shows good performance in free space and on-body scenarios. The antenna radiation pattern becomes more directive for the wearable scenario in comparison to free space which is due to the presence of the human subject. Flexibility tests have also been carried out for different bending radii corresponding to the curvature of the human limbs which are probable 
locations where the wearable devices can be placed or integrated with the clothing. The performance of the antennas deviates slightly in terms of resonant frequency, the bandwidth of the operation which is attributed to the bending condition and body effects leading to variation in the results. The antennas perform well in free space and on-body scenarios which make them suitable candidates for wearable communication applications.

\section{Data Availability}

Data are available upon request to Dr. Richa Bharadwaj (e-mail: richab@care.iitd.ac.in).

\section{Conflicts of Interest}

The authors declare that they have no conflicts of interest.

\section{Acknowledgments}

The authors would like to acknowledge the Defence Research and Development Organisation (RP03437G) for providing funding for this work.

\section{References}

[1] F. John Dian, R. Vahidnia, and A. Rahmati, "Wearables and the internet of things (IoT), applications, opportunities, and challenges: a survey," IEEE Access, vol. 8, pp. 69200-69211, 2020.

[2] M. El Gharbi, R. Fernández-García, S. Ahyoud, and I. Gil, “A review of flexible wearable antenna sensors: design, fabrication methods, and applications," Materials, vol. 13, no. 17, p. 3781, 2020.

[3] S. G. Kirtania, A. W. Elger, M. R. Hasan et al., "Flexible antennas: a review," Micromachines, vol. 11, no. 9, p. 847, 2020.

[4] M. E. Jalil, M. K. A. Rahim, N. A. Samsuri, N. A. Murad, N. Othman, and H. A. Majid, "On-body investigation of dual band diamond textile antenna for wearable applications at $2.45 \mathrm{GHz}$ and $5.8 \mathrm{GHz}$," in Proceedings of the 2013 7th European Conference on Antennas and Propagation (EuCAP), pp. 414-417, Gothenburg, Sweden, April 2013.

[5] R. Bharadwaj, S. Swaisaenyakorn, C. G. Parini, J. C. Batchelor, and A. Alomainy, "Impulse radio ultra-wideband communications for localization and tracking of human body and limbs movement for healthcare applications," IEEE Transactions on Antennas and Propagation, vol. 65, no. 12, pp. 7298-7309, 2017.

[6] H. R. Khaleel, H. M. Al-Rizzo, D. G. Rucker, and S. Mohan, "A compact polyimide-based UWB antenna for flexible electronics," IEEE Antennas and Wireless Propagation Letters, vol. 11, pp. 564-567, 2012.

[7] A. Yadav, V. Kumar Singh, A. Kumar Bhoi, G. Marques, B. Garcia-Zapirain, and I. de la Torre Díez, "Wireless body area networks: UWB wearable textile antenna for telemedicine and mobile health systems," Micromachines, vol. 11, no. 6 , p. $558,2020$.

[8] M. Ur-Rehman, T. Kalsoom, N. A. Malik et al., "A wearable antenna for mmWave IoT applications," in Proceedings of the 2018 IEEE International Symposium on Antennas and Propagation \& USNC/URSI National Radio Science Meeting, pp. 1211-1212, Boston, MA, USA, July 2018.
[9] J. Pourahmadazar and T. A. Denidni, " $60 \mathrm{GHz}$ antenna array for millimeter-wave wireless sensor devices using silver nanoparticles ink mounted on a flexible polymer substrate," Microwave and Optical Technology Letters, vol. 59, no. 11, pp. 2830-2835, 2017.

[10] R. Bharadwaj and S. K. Koul, "Assessment of limb movement activities using wearable ultra-wideband technology," IEEE Transactions on Antennas and Propagation, vol. 69, no. 4, pp. 2316-2325, 2021.

[11] M. Ullah, M. Islam, T. Alam, and F. Ashraf, "Paper-based flexible antenna for wearable telemedicine applications at $2.4 \mathrm{GHz}$ ISM band," Sensors, vol. 18, no. 12, p. 4214, 2018.

[12] C. Hertleer, H. Rogier, L. Vallozzi, and L. Van Langenhove, "A textile antenna for off-body communication integrated into protective clothing for firefighters," IEEE Transactions on Antennas and Propagation, vol. 57, no. 4, pp. 919-925, 2009.

[13] P. M. Potey and K. Tuckley, "Design of wearable textile antenna with various substrate and investigation on fabric selection," in Proceedings of the 2018 3rd International Conference on Microwave and Photonics (ICMAP), pp. 1-2, Dhanbad, India, February 2018.

[14] A. Tsolis, W. Whittow, A. Alexandridis, and J. Vardaxoglou, "Embroidery and related manufacturing techniques for wearable antennas: challenges and opportunities," Electronics, vol. 3, no. 2, pp. 314-338, 2014.

[15] A. Kiourti and J. L. Volakis, "High-geometrical-accuracy embroidery process for textile antennas with fine details," IEEE Antennas and Wireless Propagation Letters, vol. 14, pp. 1474-1477, 2015.

[16] T. Kaufmann, I. Fumeaux, and C. Fumeaux, "Comparison of fabric and embroidered dipole antennas," in Proceedings of the 2013 7th European Conference on Antennas and Propagation (EuCAP), pp. 3252-3255, Gothenburg, Sweden, April 2013.

[17] B. Ivsic, D. Bonefacic, and J. Bartolic, "Considerations on embroidered textile antennas for wearable applications," IEEE Antennas and Wireless Propagation Letters, vol. 12, pp. 1708-1711, 2013.

[18] Y. Sun, Y. Lei, Y. Liu et al., "Design of RFID textile dipole antenna," in Proceedings of the 2018 Cross Strait Quad-Regional Radio Science and Wireless Technology Conference (CSQRWC), pp. 1-2, Xuzhou, China, July 2018.

[19] M. M. Bait-Suwailam, I. I. Labiano, and A. Alomainy, "Impedance enhancement of textile grounded loop antenna using high-impedance surface (HIS) for healthcare applications," Sensors, vol. 20, no. 14, p. 3809, 2020.

[20] M. Shahidul Islam, M. T. Islam, M. A. Ullah, G. Kok Beng, N. Amin, and N. Misran, "A modified meander line microstrip patch antenna with enhanced bandwidth for $2.4 \mathrm{GHz}$ ISM-band internet of things (IoT) applications," IEEE Access, vol. 7, pp. 127850-127861, 2019.

[21] M. Mantash, A.-C. Tarot, S. Collardey, and K. Mahdjoubi, "Investigation of flexible textile antennas and AMC reflectors," International Journal of Antennas and Propagation, vol. 2012, Article ID 236505, 10 pages, 2012.

[22] A. Y. I. Ashyap, Z. Z. Abidin, S. H. Dahlan et al., "Compact and low-profile textile EBG-based antenna for wearable medical applications," IEEE Antennas and Wireless Propagation Letters, vol. 16, pp. 2550-2553, 2017.

[23] M. E. Lajevardi and M. Kamyab, "Ultraminiaturized metamaterial-inspired SIW textile antenna for off-body applications," IEEE Antennas and Wireless Propagation Letters, vol. 16, pp. 3155-3158, 2017.

[24] BERNINA-720,https:/www.bernina.com/en-IN/MachinesIN/ S\%20eries-Overview/BERNINA-7-Series/BERNINA-720. 
[25] C. Gabriel, "Compilation of the dielectric properties of body tissues at RF and microwave frequencies," Brooks Air Force, San Antonio, TX, USA, AL/OE-TR-1996-0037, 1996.

[26] S. Yan, V. Volskiy, and G. A. E. Vandenbosch, "Compact dual-band textile PIFA for $433 \mathrm{MHz} / 2.4 \mathrm{GHz}$ ISM bands," IEEE Antennas and Wireless Propagation Letters, vol. 16, pp. 2436-2439, 2017.

[27] R. Bharadwaj, C. Parini, and A. Alomainy, "Experimental investigation of 3-D human body localization using wearable ultra-wideband antennas," IEEE Transactions on Antennas and Propagation, vol. 63, no. 11, pp. 5035-5044, 2015.

[28] R. Bharadwaj and S. K. Koul, "Experimental analysis of ultrawideband body-to-body communication channel characterization in an indoor environment," IEEE Transactions on Antennas and Propagation, vol. 67, no. 3, pp. 1779-1789, 2019.

[29] S. Sankaralingam and B. Gupta, "Development of textile antennas for body wearable applications and investigations on their performance under bent conditions," Progress in Electromagnetics Research B, vol. 22, pp. 53-71, 2010.

[30] D. Ferreira, P. Pires, R. Rodrigues, and R. F. S. Caldeirinha, "Wearable textile antennas: examining the effect of bending on their performance," IEEE Antennas and Propagation Magazine, vol. 59, no. 3, pp. 54-59, 2017.

[31] A. Kavitha and J. N. Swaminathan, "Design of flexible textile antenna using FR4, jeans cotton and teflon substrates," Microsystem Technologies, vol. 25, pp. 1311-1320, 2019.

[32] P. Balaji and R. Narmadha, "Wearable E-shaped textile antenna for biomedical telemetry," in Proceedings of the 2021 International Conference on Advances in Electrical, Computing, Communication and Sustainable Technologies (ICAECT), pp. 1-5, Bhilai, India, February 2021.

[33] G. Atanasova and N. Atanasov, "Small antennas for wearable sensor networks: impact of the electromagnetic properties of the textiles on antenna performance," Sensors, vol. 20, no. 18, p. $5157,2020$.

[34] R. Sanchez-Montero, P. L. Lopez-Espi, C. Alen-Cordero, and J. A. Martinez-Rojas, "Bend and moisture effects on the performance of a U-shaped slotted wearable antenna for offbody communications in an industrial scientific medical (ISM) 2.4 GHz band," Sensors, vol. 19, no. 8, p. 1804, 2019.

[35] E. F. Sundarsingh and V. S. Ramalingam, "Design and experimental evaluation of a novel on-body textile antenna for unicast applications," Microwave and Optical Technology Letters, vol. 62, pp. 789-799, 2020.

[36] K. Masrakin, H. A. Rahim, P. J. Soh et al., "Assessment of worn textile antennas' exposure on the physiological parameters and well-being of adults," IEEE Access, vol. 7, pp. 98946-98958, 2019.

[37] H. I. Azeez, H.-C. Yang, and W.-S. Chen, "Wearable triband E-shaped dipole antenna with low SAR for IoT applications," Electronics, vol. 8, no. 6, p. 665, 2019.

[38] K. N. Paracha, S. K. Abdul Rahim, P. J. Soh, and M. Khalily, "Wearable antennas: a review of materials, structures, and innovative features for autonomous communication and sensing," IEEE Access, vol. 7, pp. 56694-56712, 2019. 